\title{
Secondary Cervicothoracic Scoliosis in Congenital Muscular Torticollis
}

\author{
Jun-Ho Kim, MD, Tae-Hoon Yum, MD, Jong Sup Shim, MD \\ Department of Orthopedic Surgery, Samsung Medical Center, Sungkyunkwan University School of Medicine, Seoul, Korea
}

Background: Although secondary cervicothoracic scoliosis frequently occurs in patients with congenital muscular torticollis (CMT), the relationship between scoliosis and CMT has not been evaluated. This study aims to evaluate the effects of surgical release of sternocleidomastoid (SCM) muscle on secondary cervicothoracic scoliosis in patients with CMT and determine factors affecting the improvement of scoliosis after surgical release of SCM muscle.

Methods: Eighty-seven of the 106 patients, confirmed as having secondary cervicothoracic scoliosis with CMT with a minimum 1-year follow-up, were included in this study. Preoperative and last follow-up radiologic outcomes were assessed for the cervicomandibular angle (CMA), Cobb angle of the cervicothoracic scoliosis, and direction of convexity in the scoliosis curve. Patients were divided into two groups to assess the improvement of Cobb angle according to residual growth potential; age $\leq 15$ years and $>15$ years. The improvement of Cobb angle after surgical release was compared in the two groups. Correlation analysis and multivariable regression analysis were performed to determine the factors affecting the improvement of scoliosis.

Results: All the radiologic parameters, such as the Cobb angle and CMA, improved significantly after surgical release $(p<0.001)$. The improvement of Cobb angle was significantly higher in age $\leq 15$ years than in age $>15$ years $(p<0.001)$. The improvement of Cobb angle was significantly correlated with age $(r=-0.474, p<0.001)$ and the preoperative Cobb angle $(r=0.221, p=0.036)$. In multivariable regression analysis, age and preoperative Cobb angle were shown to be predisposing factors affecting the improvement of scoliosis.

Conclusions: The results showed that SCM release can be a beneficial treatment for secondary cervicothoracic scoliosis. The improvement of scoliosis was greater when the SCM release was performed before the patient reached the end of growth.

Keywords: Cervicomandibular angle, Congenital muscular torticollis, Cobb angle, Secondary scoliosis, Sternocleidomastoid release

Torticollis is derived from a Latin word meaning twisted neck. ${ }^{1,2)}$ Congenital muscular torticollis (CMT) is one of the most common types of this condition and is caused by fibromatous contracture of the sternocleidomastoid (SCM) muscle. ${ }^{2-4)}$ It usually presents with a hard mass within the affected SCM muscle in the neonatal

Received January 30, 2019; Revised March 4, 2019; Accepted April 10, 2019

Correspondence to: Jong Sup Shim, MD

Department of Orthopedic Surgery, Samsung Medical Center, Sungkyunkwan University School of Medicine, 81 Irwon-ro, Gangnam-gu, Seoul 06351, Korea

Tel: +82-2-3410-3505, Fax: +82-2-3410-0061

E-mail: jss3505@skku.edu period. ${ }^{1,3,5,6)}$ As the mass gradually subsides with time, the affected muscle is shortened and becomes a fibrotic band. ${ }^{1,3,5,6)}$ Contracture of the SCM muscle leads to an ipsilateral tilt of the head with a contralateral rotation of the face, resulting in typical symptoms, such as limited range of motion (ROM) of the neck. ${ }^{3,5,6)}$ Early diagnosis and physiotherapy is necessary to improve the deformity. ${ }^{6,7)}$ If untreated, the prolonged twisted position of the neck may lead to compensatory changes of adjacent skeletal structures, resulting in both craniofacial asymmetry and secondary cervicothoracic scoliosis. ${ }^{1,4,5,8)}$

A number of studies have shown the relationship between craniofacial asymmetry and CMT from different perspectives. ${ }^{3-5)}$ Although the scoliosis in CMT is 
Kim et al. Cervicothoracic Scoliosis in Congenital Torticollis

Clinics in Orthopedic Surgery • Vol. 11, No. 3, $2019 \bullet$ www.ecios.org

secondary scoliosis, this long-standing scoliosis can still lead to the risk of radiologic exposure during the followup period, cosmetic dissatisfaction, and the possibility of degenerative spondylitis. ${ }^{9-12)}$ However, little is known about the relationship between CMT and secondary scoliosis, and no study has mainly focused on scoliosis in patients with CMT. In the previous few studies on the outcome of patients after surgical treatment of CMT, scoliosis was rarely included in the list of parameters to be evaluated. ${ }^{2,6,8)}$ In addition, the evaluation of scoliosis was limited to changes after surgical treatment and the number of patients in these studies was limited. ${ }^{2,6,8)}$ Therefore, our study focused on the development of scoliosis in patients with CMT, and the changes that might occur to the scoliosis after surgical release in these patients.

We sought to determine (1) the prevalence of secondary scoliosis in patients with CMT and its relation to CMT, in terms of direction and severity, (2) improvement of secondary scoliosis after surgical release in patients with CMT, (3) difference in the improvement of secondary scoliosis between patients still growing and fully grown, and (4) factors affecting improvement of secondary scoliosis after surgical release in patients with CMT.

\section{METHODS}

\section{Study Design and Patients}

We retrospectively reviewed the medical records of patients who had a surgical release of the SCM muscle (SCM release) for CMT between March 2007 and September 2015 at Samsung Medical Center. One hundred twentythree patients with CMT underwent SCM release. The exclusion criteria were (1) age between 1 and 4 years, (2) torticollis of unknown etiology or neurologic abnormality, (3) congenital osseous torticollis, (4) history of SCM release on the same side, (5) a Cobb angle of $<10^{\circ}$ or scoliotic curvature starting at middle thoracic level since this does not meet the criteria for cervicothoracic scoliosis, ${ }^{13,14)}$ and (6) less than 1-year follow-up. The excluded patients were two patients between 1 and 4 years of age; one patient with underlying neurofibromatosis; four patients with previous surgery on the affected side; 18 patients with a Cobb angle of $<10^{\circ}$; and one patient with scoliotic curvature starting at T8 level. In the remaining 97 patients, preoperative radiography was performed for evaluation. However, 10 patients were lost to follow-up in less than 1 year; thus, 87 patients were enrolled as subjects in this study. To verify the association between age and improvement of the scoliosis, patients were divided
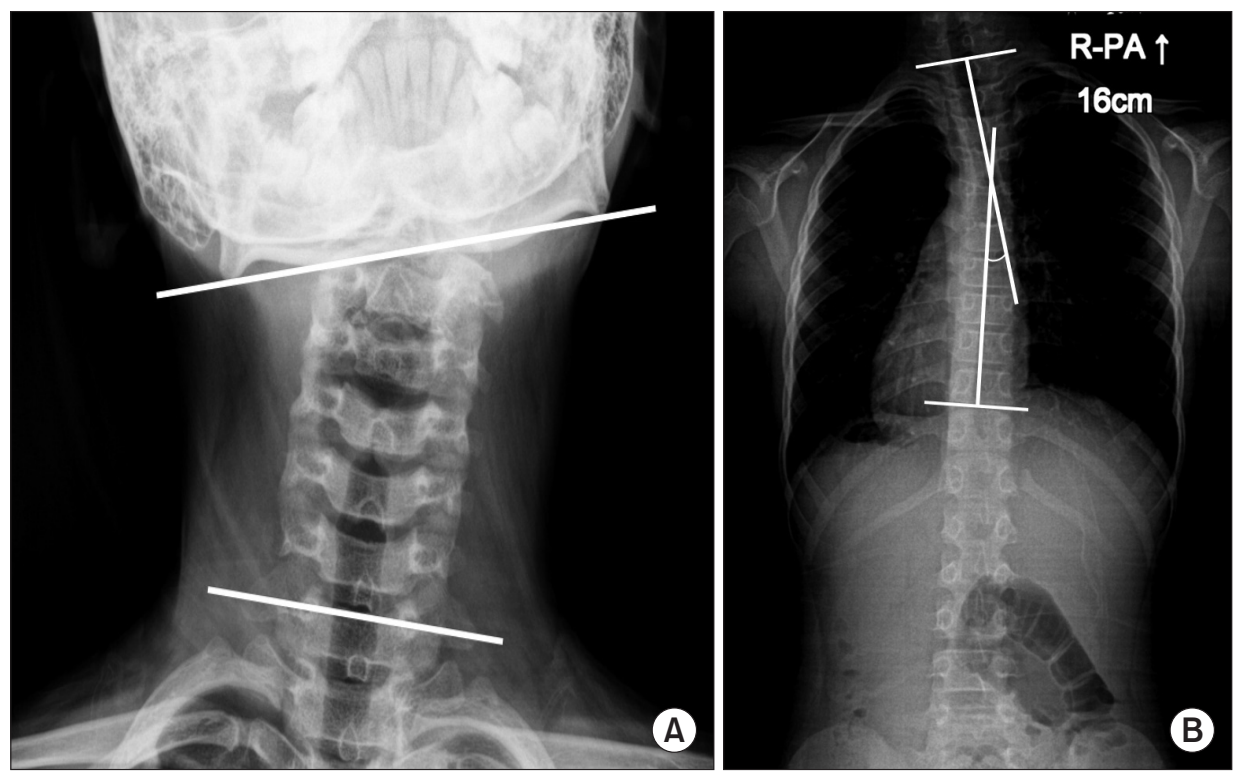

Fig. 1. (A) The cervicomandibular angle (CMA) was measured on the anteroposterior radiograph of the cervical spine to quantify the degree of head tilt. The CMA was defined as the angle between a line connecting the lower margins of the mandibular angles (upper line) and a line drawn along the upper border of the C7 vertebral body (lower line). ${ }^{6,15)}$ (B) Cobb angle was measured on the posteroanterior (PA) radiograph of the whole spine to quantify the magnitude of scoliosis. The Cobb angle was defined as the angle formed by two perpendicular lines between the superior endplate (upper line) of the proximal end vertebra and the inferior endplate of the distal end vertebra (lower line). ${ }^{16)}$ 
Kim et al. Cervicothoracic Scoliosis in Congenital Torticollis

Clinics in Orthopedic Surgery • Vol. 11, No. 3, $2019 \bullet$ www.ecios.org

into two groups according to the age at the time of operation. We divided the two groups at the age of 15 years, approximately the end of the growth period, to assess the improvement of the scoliosis. The minimum follow-up was 12 months and the mean follow-up was 28.1 months (range, 12 to 99 months). The Institutional Review Board of Samsung Medical Center (IRB No. 2016-11-148-001) approved this retrospective study and waived the requirement for informed patient consent.

\section{Surgical Protocol and Postoperative Rehabilitation}

A senior surgeon (JSS) performed a uniform surgical procedure of either a unipolar or a bipolar release. The type of surgical procedure was determined intraoperatively to achieve complete release. Bipolar release was done when manipulation could not provide proper correction after unipolar release. The surgical procedures were performed using a specific surgical technique described previously. ${ }^{6,15)}$ A brace was then applied for 6 to 8 hours a day for about 6 weeks to maintain a slightly overcorrected position. The patients were advised to perform stretching exercises for at least 10 minutes four to six times per day.

\section{Variables and Outcomes}

All patients were evaluated preoperatively by the senior author (JSS). The motion deficit of passive neck ROM, including rotation and lateral bending, was calculated by comparing with the neck ROM of the contralateral side with a goniometer. The difference in the chin-to-eyelid distance was measured using a ruler for the assessment of facial asymmetry. Radiographic evaluations were performed by two independent examiners. The preoperative and the latest follow-up plain radiographs were evaluated. The cervicomandibular angle (CMA) was measured from an anteroposterior radiograph of the cervical spine to quantify the degree of head tilt. ${ }^{6,15)}$ The CMA was defined as the angle between a line connecting the lower margins of the mandibular angles and a line drawn along the upper border of the C7 vertebral body (Fig. 1). ${ }^{6,15}$ The Cobb angle of the cervicothoracic spine was measured from a posteroanterior standing radiograph of the whole spine to quantify the severity of the scoliosis (Fig. 1). The direction of the curve convexity of the scoliosis was evaluated from a whole spine posteroanterior radiograph (Fig. 1).

\section{Statistical Analysis}

Using these methods of evaluation, four steps of analysis were carried out. The first step was to determine the prevalence of scoliosis in patients with CMT who had a Cobb angle of $>10^{\circ}$ preoperatively among all the patients with CMT who met the inclusion criteria. Correlation analyses were used to assess the relationship between the preoperative Cobb angle and other variables. In addition, multivariable regression analysis was performed to identify factors affecting the preoperative Cobb angle by means of the severity of scoliosis. In the second step, paired $t$-test and Wilcoxon signed-rank tests were used to compare the radiographic parameters before and after surgery in this cohort. In the third step, two sample $t$-test and Wilcoxon signed-rank tests were used to compare the radiographic parameters between age groups before and after surgery. The fourth step was to assess the relationship between improvement of the Cobb angle and other variables, using correlation analyses. In addition, multivariable regression analyses were performed to identify factors affecting the improvement of the Cobb angle. Statistical analyses were performed using the SAS ver. 9.4 (SAS Institute Inc., Cary, NC, USA). A p-value of $<0.05$ was considered significant.

Table 1. Patient Characteristics

\begin{tabular}{lc}
\hline \multicolumn{1}{|c}{ Variable } & Value $(\mathrm{n}=87)$ \\
\hline Age $(\mathrm{yr})$ & $15.8 \pm 11.7(5-44)$ \\
\hline Sex & 46 \\
\hline Male & 41 \\
\hline Female & 57 \\
\hline Affected side & 30 \\
\hline Right & $5.3 \pm 4.0(0-26)$ \\
\hline Left & \\
\hline Chin-to-eyelid distance $(\mathrm{mm})^{*}$ & $30.8 \pm 14.8(0-70)$ \\
\hline Motion deficit & \\
\hline Lateral bending $\left(^{\circ}\right)$ & $21.7 \pm 11.9(0-60)$ \\
\hline Rotation $\left({ }^{\circ}\right)$ & 66 \\
\hline Surgical method & 21 \\
\hline Unipolar & \\
\hline Bipolar & \\
\hline
\end{tabular}

Values are presented as mean \pm standard deviation (range). *Distance of unaffected side minus distance of affected side. ${ }^{\dagger}$ Motion of unaffected side minus motion of affected side. 
Kim et al. Cervicothoracic Scoliosis in Congenital Torticollis

Clinics in Orthopedic Surgery • Vol. 11, No. 3, 2019 • www.ecios.org
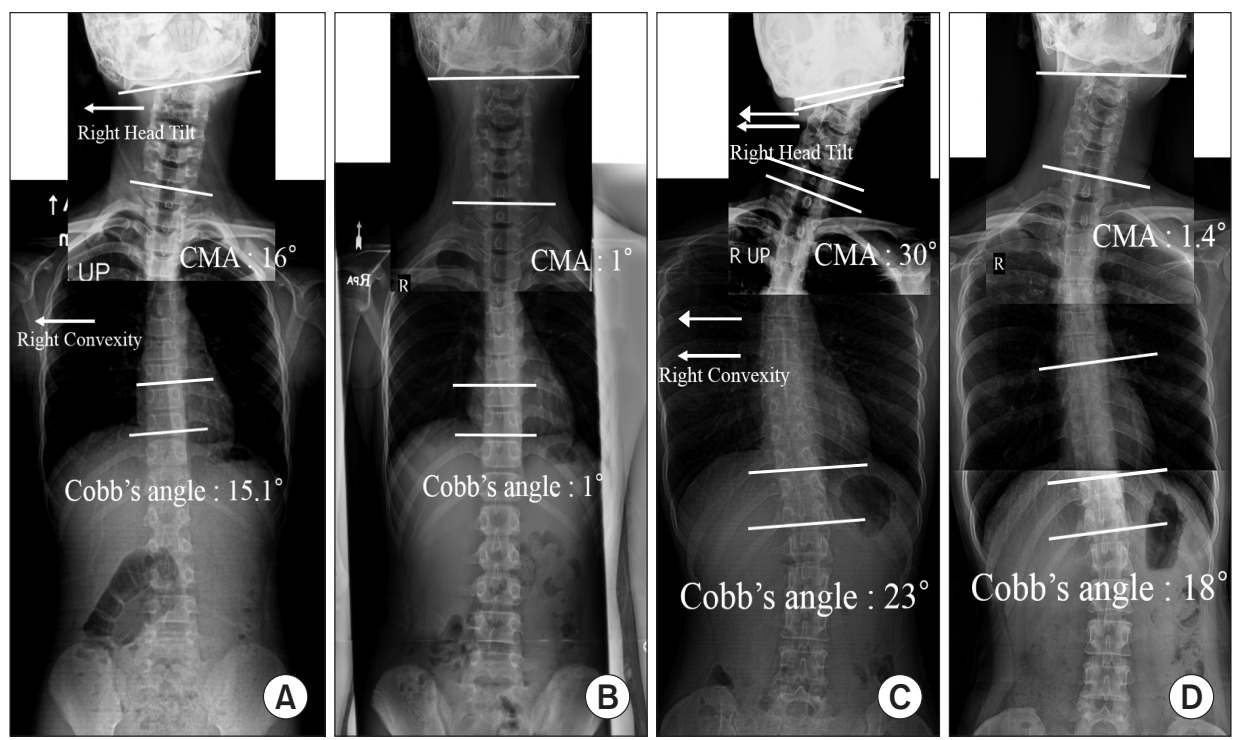

Fig. 2. An anteroposterior radiograph of the cervical spine was attached to a posteroanterior radiograph of the whole spine to show the coronal deformity of the axial skeletal structure not only with scoliosis but also with head tilt. (A, B) A 9-year-old girl presented with congenital muscular torticollis. (A) The preoperative plain radiograph shows a $16^{\circ}$ cervicomandibular angle (CMA) and a $15.1^{\circ}$ Cobb angle. The direction of head tilt was identical to the direction of the convexity of the curvature. (B) Twenty months after unipolar sternocleidomastoid (SCM) muscle release, the patient showed improvement of the head tilt and scoliosis. Her CMA improved to $1^{\circ}$ and her Cobb angle decreased to $1^{\circ}$. (C, D) A 30-year-old woman presented with neglected congenital muscular torticollis. (C) The preoperative plain radiograph shows a $30^{\circ} \mathrm{CMA}$ and a $23^{\circ}$ Cobb angle. The direction of head tilt was identical to the direction of the convexity of the curvature. (D) Thirty-one months after bipolar SCM muscle release, the patient showed improvement of the head tilt and scoliosis. Her CMA improved to $1.4^{\circ}$ and her Cobb angle decreased to only $18^{\circ}$.

\section{RESULTS}

Patients' demographic characteristics and preoperative data are shown in Table 1.

\section{Prevalence of Secondary Scoliosis and Its Relation to CMT}

Eighty-seven patients with cervicothoracic scoliosis were identified out of 106 patients with CMT. The prevalence of secondary scoliosis in patients with CMT was $82.1 \%$. The tilting side of the head in CMT was matched with the direction of convexity in the scoliosis curve (Fig. 2). Moreover, the preoperative Cobb angle increased proportionally to the preoperative CMA $(r=0.440, p<0.001)$ in the simple correlation analysis. The multivariable regression analysis demonstrated that preoperative CMA was a significant predisposing factor for the preoperative Cobb angle in patients with CMT (Table 2).

\section{Improvement of Secondary Scoliosis after SCM Release in CMT}

The mean preoperative CMA was $16.0^{\circ}$ (range, $2.1^{\circ}$ to $37.3^{\circ}$ ) and the mean preoperative Cobb angle was $15.5^{\circ}$ (range, $10.5^{\circ}$ to $31.7^{\circ}$ ); these values were reduced to a mean of $5.1^{\circ}$ (range, $-0.6^{\circ}$ to $27.7^{\circ}$ ) and $7.4^{\circ}$ (range, $0.2^{\circ}$

\begin{tabular}{|c|c|c|c|}
\hline Variable & Level & Coefficient & $\begin{array}{c}\text { Standard } \\
\text { error }\end{array}$ \\
\hline Intercept & & 12.545 & 1.362 \\
\hline Age & & -0.084 & 0.042 \\
\hline Sex & Male vs. female & -1.849 & 0.879 \\
\hline Affected side & Left vs. right & 0.008 & 0.862 \\
\hline Chin-to-eyelid difference & & -0.158 & 0.112 \\
\hline Lateral bending deficit & & 0.046 & 0.037 \\
\hline Rotation deficit & & -0.093 & 0.043 \\
\hline Preoperative CMA & & 0.303 & 0.068 \\
\hline
\end{tabular}

The coefficient indicates the change in the percentage of one group relative to the reference group (for categorical variables) or the change resulting from one unit increase of the input variable (for continuous variables). Statistical significance was set at a $p$-value of $<0.05$. CMA: cervicomandibular angle.

to $23.7^{\circ}$ ), respectively, at the latest follow-up. All of the radiologic parameters, including the Cobb angle and CMA, improved significantly after SCM release $(p<0.001)$. 
Kim et al. Cervicothoracic Scoliosis in Congenital Torticollis

Clinics in Orthopedic Surgery • Vol. 11, No. 3, $2019 \bullet$ www.ecios.org

\section{How the Improvement of Secondary Scoliosis Is Different between Patients Still Growing and Full-Grown}

The mean Cobb angle reduced significantly postoperatively in both age groups $(p<0.001)$. Improvement of the Cobb angle in the group of age $\leq 15$ years was significantly greater than that in the group of age $>15$ years $(p$ $<0.001$ ) (Table 3).

Factors Affecting Improvement of Secondary Scoliosis after SCM Release in Patients with CMT

Improvement of the Cobb angle after SCM release decreased proportionally to age $(r=-0.474, p<0.001)$ and increased proportionally to the preoperative Cobb angle $(r=0.221, p=0.036)$ in the simple correlation analysis. The multivariable regression analysis demonstrated that age and the preoperative Cobb angle were significant predisposing factors for improvement of the Cobb angle in patients with CMT (Table 4).

\section{DISCUSSION}

The main finding of our study is that we investigated the prevalence of secondary scoliosis in patients with CMT and determined whether the direction or severity of CMT is associated with that of scoliosis. Our study also investigated whether SCM release was useful in treating secondary scoliosis, as well as CMT, and how improvement of secondary scoliosis differed between patients still growing and full-grown. In addition, we investigated factors affecting improvement of secondary scoliosis.

The prevalence of secondary cervicothoracic scoliosis in patients with CMT was $82.1 \%$ in our study. Although craniofacial asymmetry has been reported in

\section{Table 3. Changes of Cobb Angle According to the Age at the Time of Surgery}

\begin{tabular}{|c|c|c|c|c|}
\hline Variable & Age $\leq 15$ yr $(n=55)$ & Age $>15$ yr $(n=32)$ & $95 \% \mathrm{Cl}$ of difference & $p$-value \\
\hline Preoperative Cobb angle $\left({ }^{\circ}\right)$ & $15.4 \pm 4.1$ & $15.7 \pm 4.6$ & -02.2 to 1.6 & 0.762 \\
\hline Last follow-up Cobb angle $\left({ }^{\circ}\right)$ & $5.5 \pm 3.5$ & $10.6 \pm 5.5$ & -07.2 to -02.9 & $<0.001$ \\
\hline Improvement Cobb angle $\left({ }^{\circ}\right)$ & $9.9 \pm 3.6$ & $5.2 \pm 3.9$ & 3.1 to 6.4 & $<0.001$ \\
\hline
\end{tabular}

Values are presented as mean \pm standard deviation.

Cl: confidence interval.

Table 4. Multivariable Regression Analysis for the Improvement of Cobb Angle

\begin{tabular}{|c|c|c|c|c|c|}
\hline Variable & Level & Coefficient & Standard error & T statistic & $p$-value \\
\hline Intercept & & 11.857 & 1.426 & 8.32 & $<0.001$ \\
\hline Age & & -0.213 & 0.034 & -5.16 & $<0.001$ \\
\hline Sex & Male vs. female & -0.111 & 0.909 & -0.12 & 0.904 \\
\hline Affected side & Left vs. right & 0.116 & 0.894 & 0.13 & 0.898 \\
\hline Chin-to-eyelid difference & & 0.053 & 0.099 & 0.54 & 0.594 \\
\hline Lateral bending deficit & & 0.003 & 0.033 & 0.08 & 0.934 \\
\hline Rotation deficit & & -0.049 & 0.040 & -1.55 & 0.212 \\
\hline Preoperative Cobb angle & & 0.403 & 0.093 & 4.32 & $<0.001$ \\
\hline Operative technique & Bipolar vs. unipolar & 0.444 & 1.029 & 0.43 & 0.667 \\
\hline Improvement of CMA & & 0.038 & 0.080 & 0.47 & 0.632 \\
\hline
\end{tabular}

The coefficient indicates the change in the percentage of one group relative to the reference group (for categorical variables) or the change resulting from one unit increase of the input variable (for continuous variables). Statistical significance was set at a $p$-value of $<0.05$.

CMA: cervicomandibular angle. 
Kim et al. Cervicothoracic Scoliosis in Congenital Torticollis

Clinics in Orthopedic Surgery • Vol. 11, No. 3, $2019 \bullet$ www.ecios.org

$90.1 \%$ of patients operated on for $\mathrm{CMT}^{17)}$ it is the first report, to our knowledge, regarding the prevalence of secondary scoliosis in patients with CMT who underwent SCM release. As development of craniofacial asymmetry is known to be attributable to $\mathrm{CMT}^{3-5)}$ we believe that cervicothoracic scoliosis is also caused by CMT. Because of the spine's ability to compensate for postural imbalance in the axial skeleton, the presence of a compensatory lumbar curvature in the setting of significant leg-length discrepancy is well established. ${ }^{18-20)}$ In our study, we demonstrated that the tilting side of the head was identical to the direction of convexity in the scoliosis curve, and the preoperative CMA was a significant factor affecting the preoperative Cobb angle. Although an obvious relationship is unclear, the correlation between CMT and scoliosis severity and convexity suggests that the two entities are related in these patients.

We found that SCM release is beneficial to secondary cervicothoracic scoliosis in CMT and that the Cobb angle was significantly reduced from $15.5^{\circ}$ to $7.4^{\circ}$. This effective change might be helpful because long-standing scoliosis could lead to several problems, such as degenerative spondylitis or clinical symptoms, although some studies do not agree. ${ }^{7,21)}$ Recently, Mesfin et al. ${ }^{22)}$ reported a 54-year-old woman with cervicothoracic scoliosis and CMT, who underwent a spinal fusion due to degenerative arthritis and pain. Previous studies found the long-term outcome of a patient with scoliosis who had a higher possibility of degenerative changes and pain compared with a patient without scoliosis. ${ }^{21,23,24)}$

The optimal timing of surgery for CMT remains controversial. Previous studies have stated that an early SCM release can be helpful for improving craniofacial asymmetry before the age of 5 years. ${ }^{3,5,7,17,25)}$ In contrast, many studies recommended that the surgery should be delayed until the patient can comply with postoperative rehabilitation and showed satisfactory clinical outcomes. $^{6,8,12,15,26)}$ However, to our knowledge, there was no study focusing on secondary scoliosis in terms of the optimal timing of surgery for CMT. We found the improvement of secondary scoliosis in the older patient group (age $>15$ years) was significantly less than that in the younger patient group (age $\leq 15$ years). Furthermore, residual deformity of scoliosis after surgery was about $10^{\circ}$ in the older patient group (age $>15$ years). We believe that young patients have a greater potential for remodeling the scoliosis after surgery and long-standing cases may progress toward a fixed deformity. Using threedimensional computed tomography, Hussein et al. ${ }^{2)}$ demonstrated that there were definitive vertebral deformities along the cervical spine in long-standing patients with CMT. Therefore, we suggest that it may be better to perform surgery for patients with CMT and scoliosis when growth potential remains.

Improvement of secondary scoliosis in patients with CMT was significantly affected by age and the preoperative Cobb angle in our regression analysis. Although many studies reported the relation between age and improvement of craniofacial asymmetry, ${ }^{3-5)}$ this study was the first study to show the effect of age on improvement of secondary scoliosis in CMT. Improvement in scoliosis was significantly affected by the preoperative severity of scoliosis, according to the regression analysis. This result indicates that cervicothoracic vertebrae with more severe scoliosis has a more potential angle to be neutral from the tethered position than those with less severe scoliosis. However, this result does not definitely mean that the scoliosis resolves with lower age and higher preoperative severity, because this is a retrospective observational study.

There are several limitations of our study. First, it was a retrospective case series without a control group and had a nonrandomized study design. This study design did not allow us to assess perfectly how effective the surgery was for scoliosis compared with conservative treatment, because no patients were treated nonoperatively. Second, some readers may think that the present study has a short-term follow-up where patients were evaluated only for a minimum of 1 year after surgery. However, the minimum 1-year follow-up is long enough to evaluate the status after SCM release, because the entire process of soft tissue maturation usually takes less than one year. ${ }^{8)}$ Third, the number of patients in this study was small. However, the author tried to follow up all patients, and routine radiographs of the whole spine only began in patients with CMT a few years ago. Fourth, we only evaluated a single radiographic parameter of either CMT or scoliosis to quantify the deformity. Since both the CMA and Cobb angle represent degrees of coronal deformity, parameters demonstrating rotational deformity should have been added to the evaluation. Fifth, the clinical outcome concerning scoliosis was not included in this study. Because patient-derived outcomes have become increasingly important, we should include validated clinical outcomes regarding scoliosis in future. However, we believe that the evaluations of only objective outcomes in this study are also meaningful.

Secondary cervicothoracic scoliosis frequently occurred in patients with CMT, particularly in those who needed surgical treatment, and correlated with CMT in 
Kim et al. Cervicothoracic Scoliosis in Congenital Torticollis

Clinics in Orthopedic Surgery • Vol. 11, No. 3, $2019 \bullet$ www.ecios.org

terms of direction and severity. We confirmed that SCM release is a beneficial treatment for not only CMT but also scoliosis. Improvement of scoliosis was greater when surgery was performed before the patient reached 15 years of age, and there was a more potential for remodeling scoliosis in younger patients who were still growing. In conclusion, age and preoperative Cobb angle were sig- nificant factors affecting improvement of scoliosis after SCM release in patients with CMT.

\section{CONFLICT OF INTEREST}

No potential conflict of interest relevant to this article was reported.

\section{REFERENCES}

1. Ta JH, Krishnan M. Management of congenital muscular torticollis in a child: a case report and review. Int J Pediatr Otorhinolaryngol. 2012;76(11):1543-6.

2. Hussein MA, Yun IS, Park H, Kim YO. Cervical spine deformity in long-standing, untreated congenital muscular torticollis. J Craniofac Surg. 2017;28(1):46-50.

3. Lee JK, Moon HJ, Park MS, Yoo WJ, Choi IH, Cho TJ. Change of craniofacial deformity after sternocleidomastoid muscle release in pediatric patients with congenital muscular torticollis. J Bone Joint Surg Am. 2012;94(13):e93.

4. Seo SJ, Kim JH, Joh YH, et al. Change of facial asymmetry in patients with congenital muscular torticollis after surgical release. J Craniofac Surg. 2016;27(1):64-9.

5. Seo SJ, Yim SY, Lee IJ, et al. Is craniofacial asymmetry progressive in untreated congenital muscular torticollis? Plast Reconstr Surg. 2013;132(2):407-13.

6. Lim KS, Shim JS, Lee YS. Is sternocleidomastoid muscle release effective in adults with neglected congenital muscular torticollis? Clin Orthop Relat Res. 2014;472(4):12718.

7. Canale ST, Griffin DW, Hubbard CN. Congenital muscular torticollis: a long-term follow-up. J Bone Joint Surg Am. 1982;64(6):810-6.

8. Omidi-Kashani F, Hasankhani EG, Sharifi R, Mazlumi M. Is surgery recommended in adults with neglected congenital muscular torticollis? A prospective study. BMC Musculoskelet Disord. 2008;9:158.

9. Mogaadi M, Ben Omrane L, Hammou A. Effective dose for scoliosis patients undergoing full spine radiography. Radiat Prot Dosimetry. 2012;149(3):297-303.

10. Newton PO, Khandwala Y, Bartley CE, Reighard FG, Bastrom TP, Yaszay B. New EOS imaging protocol allows a substantial reduction in radiation exposure for scoliosis patients. Spine Deform. 2016;4(2):138-44.

11. Danielsson AJ, Nachemson AL. Back pain and function 22 years after brace treatment for adolescent idiopathic scoliosis: a case-control study-part I. Spine (Phila Pa 1976). 2003;28(18):2078-85.
12. Patwardhan S, Shyam AK, Sancheti P, Arora P, Nagda T, Naik P. Adult presentation of congenital muscular torticollis: a series of 12 patients treated with a bipolar release of sternocleidomastoid and Z-lengthening. J Bone Joint Surg Br. 2011;93(6):828-32.

13. Goldstein LA, Waugh TR. Classification and terminology of scoliosis. Clin Orthop Relat Res. 1973;(93):10-22.

14. Keynan O, Fisher CG, Vaccaro A, et al. Radiographic measurement parameters in thoracolumbar fractures: a systematic review and consensus statement of the spine trauma study group. Spine (Phila Pa 1976). 2006;31(5):E15665.

15. Shim JS, Jang HP. Operative treatment of congenital torticollis. J Bone Joint Surg Br. 2008;90(7):934-9.

16. Cobb JR. Outline for the study of scoliosis. Am Acad Orthop Surg Instr Course Lect. 1948;5:261-75.

17. Cheng JC, Tang SP. Outcome of surgical treatment of congenital muscular torticollis. Clin Orthop Relat Res. 1999;(362):190-200.

18. Jones KB, Sponseller PD, Hobbs W, Pyeritz RE. Leg-length discrepancy and scoliosis in Marfan syndrome. J Pediatr Orthop. 2002;22(6):807-12.

19. Papaioannou T, Stokes I, Kenwright J. Scoliosis associated with limb-length inequality. J Bone Joint Surg Am. 1982;64(1):59-62.

20. Walker AP, Dickson RA. School screening and pelvic tilt scoliosis. Lancet. 1984;2(8395):152-3.

21. Danielsson AJ, Nachemson AL. Back pain and function 23 years after fusion for adolescent idiopathic scoliosis: a case-control study-part II. Spine (Phila Pa 1976). 2003;28(18):E373-83.

22. Mesfin A, Bakhsh WR, Chuntarapas T, Riew KD. Cervical scoliosis: clinical and radiographic outcomes. Global Spine J. 2016;6(1):7-13.

23. Fowles JV, Drummond DS, L'Ecuyer S, Roy L, Kassab MT. Untreated scoliosis in the adult. Clin Orthop Relat Res. 1978;(134):212-7. 
Kim et al. Cervicothoracic Scoliosis in Congenital Torticollis

Clinics in Orthopedic Surgery • Vol. 11, No. 3, 2019• www.ecios.org

24. Nachemson A. A long term follow-up study of non-treated scoliosis. Acta Orthop Scand. 1968;39(4):466-76.

25. Wirth CJ, Hagena FW, Wuelker N, Siebert WE. Biterminal tenotomy for the treatment of congenital muscular torticollis: long-term results. J Bone Joint Surg Am.
1992;74(3):427-34.

26. Shim JS, Noh KC, Park SJ. Treatment of congenital muscular torticollis in patients older than 8 years. J Pediatr Orthop. 2004;24(6):683-8. 